\title{
CUIDADO À SAÚDE DA PESSOA EM SITUAÇÃO DE RUA: DEBATE E RELATOS PARA UMA ABORDAGEM INTERSETORIAL
}

\section{Káren Maria Rodrigues da Costa}

Psicóloga, Residente em Saúde da Família (UFPI), Brasil.

E-mail: karen.r.costa@hotmail.com

João Janilson da Silva Sousa

Farmacêutico, Residente em Saúde da Família (UFPI), Brasil.

\section{Rebeca Barbosa da Rocha}

Fisioterapeuta, Residente em Saúde da Família (UFPI), Brasil.

\section{Maísa Ravenna Beleza Lino}

Enfermeira, Residente em Saúde da Família (UFPI), Brasil.

\section{Geovania Vieira de Brito}

Enfermeira, Mestre em Saúde da Família. Prefeitura Municipal de Parnaíba (PI), Brasil.

\section{Noé Fontenele de Sousa}

Enfermeiro, Especialista em Saúde Mental. CAPS II. Brasil
RESUMO: Este artigo apresenta relato referente ao "I Encontro sobre as Políticas de Atendimento à População em Situação de Rua: Articulação entre SUS e SUAS", realizado por residentes da Residência Multiprofissional em Saúde da Família em Parnaíba (PI) durante os meses de outubro e novembro de 2016. O objetivo é discorrer sobre o encontro e discutir as políticas de atendimento à pessoa em situação de rua - PSR. O evento teve como público-alvo os agentes comunitários de saúde (ACS) e enfermeiros de quatro Distritos de Saúde. O evento possibilitou a discussão sobre a PSR, as políticas de reconhecimento dessa população, a existência e qualidade do serviço ofertado pelo Centro de Referência Especializado para População de Rua e a discussão de casos. Desse modo, o evento proporcionou aos profissionais de saúde a oportunidade de lançar um novo olhar em relação à PSR e criou a possibilidade de realização de outros eventos com o mesmo tema destinado aos profissionais de outros setores da rede.

PALAVRAS-CHAVE: Pessoas em situação de rua; Sistema Único de Saúde; ação intersetorial.

\section{HEALTH CARE OF THE HOMELESS: DEBATE AND REPORTS FOR AN INTERSECTORIAL APPROACH}

ABSTRACT: This paper presents a report about the "I Conference on Policies for Assistance to Homeless People: Articulation between SUS and SUAS", held on October 2016 by a team of residents of the Multiprofessional Residence in Family Health in Parnaíba city, Brazil, from October to November 2016. This study aims to discuss the Conference and the assistance policies for homeless people. The conference's audience was the Health Community Agents (HCA) and nurses from 4 Health Districts. The event allowed discussions about homeless people, recognition policies of this population, existence and quality of the service offered by the Specialized Reference Centre for the Homeless People and case discussions. Thus, the health professionals on Conference had the opportunity to take a fresh look towards the homeless people and raised the possibility of the realization of other events with the same subject intended to professionals from other sectors of the network.

KEY WORDS: Homeless; Health Unic System; Intersectoral action.

\section{INTRODUÇ̃̃o}

Quem de nós não se encontra rotineiramente com a figura de uma pessoa em situação de rua (PSR)? Nas calçadas das ruas, em 
mercados públicos ou até mesmo deitada em bancos e canteiros das principais avenidas das cidades. Esse encontro cotidiano levanta a reflexão do que fazemos diante de tal individuo caso ele necessite de um acompanhamento de saúde? Como e onde encaminhálo? Como acompanhá-lo ao Serviço de Proteção Social Especial? E como garantir o acesso da PSR às ações e aos serviços de saúde e assistência social? Questões como estas necessitam serem pensadas e incorporadas na rotina dos profissionais que trabalham na "porta de entrada" dos serviços de saúde, ou seja, na Atenção Primária à Saúde. Além disso, é indispensável fortalecer a perspectiva de convívio grupal e social, autonomia, mobilização e participações sociais previstos como objetivos da Proteção Social Especial para esta população (BRASIL, 2011).

Nesta perspectiva, este, artigo discorre sobre a experiência do "I Encontro sobre as Políticas de Atendimento à População em Situação de Rua: Articulação entre SUS e SUAS", que teve como objetivo discutir as políticas de atendimento à PSR impulsionar uma articulação entre os trabalhadores das áreas da saúde e assistência social que atuam com as PSR e apresentar o Centro de Referência Especializado para PSR aos trabalhadores da saúde.

Desse modo, este evento surge de reflexões e observações realizadas no processo de territorialização e ações neste campo, evidenciando-se um distanciamento por parte de alguns profissionais no que concerne à saúde da população em situação de rua, não especificamente somente seu processo de saúde e doença, mas sim, a falta de acolhimento, demandas negligenciadas no território, desconhecimento da Política Nacional para Pessoa em Situação de Rua por parte dos profissionais. Somando-se a isto, houve solicitação por parte da equipe do Centro POP da realização de uma articulação maior com os profissionais de Atenção Básica no que se refere à assistência à saúde de seus usuários, tendo em vista as dificuldades relatadas na resolução de alguns casos acompanhados por este dispositivo da assistência social.

\section{METODOLOGIA}

Este estudo consiste em um relato descritivo de experiência com base na construção de uma articulação entre saúde e assistência social visando o cuidado em saúde da PSR. O evento - "I Encontro sobre as Políticas de Atendimento a Pessoa em Situação de Rua" teve como tema "A Articulação entre SUS e SUAS", tendo sido realizado de outubro a novembro de 2016 na Escola de Gestão em Saúde Pública e no auditório de uma Unidade Básica de Saúde (UBS)- Mód.39 (CEEP), na cidade de Parnaíba-PI.

\subsection{PARTICIPANTES}

O público participante foi composto, em média, por 159 Agentes Comunitários de Saúde (ACS) e 38 enfermeiros dos quatros Distritos de Saúde. O encontro foi realizado em diferentes momentos, sendo quatro dias destinado aos ACS de cada distrito e dois dias aos enfermeiros das Unidades Básicas de Saúde (UBS).

Quanto aos debatedores ou apresentadores, contou-se com a colaboração de um Professor Doutor da Universidade Federal do Piauí-UFPI que dialogou sobre a Saúde da População de Rua. Ademais, a equipe técnica do Centro de Referência Especializado para População em Situação de Rua (Centro POP) abordou o tema na perspectiva da assistência social. A equipe de residentes da Saúde da Família-UFPI (enfermeira, farmacêutico, fisioterapeuta e psicóloga) foram os articuladores e organizadores do evento.

\subsection{PROCEDIMENTOS}

A ideia do evento surgiu a partir de discussões entre profissionais do Centro POP e residentes do Programa de Residência Multiprofissional em Saúde da Família da Universidade Federal do Piauí (UFPI). Durante o processo de territorialização, os residentes identificaram casos de PSR vivendo em condições precárias de saúde. Eles reportaram-se ao Centro POP, único dispositivo de referência implantado no município para a PSR, com o objetivo de procurar conhecer esta população e a forma como realizava-se a articulação na rede com a saúde.

Durante as discussões e observando a rotina de algumas unidades de saúde, a equipe de residentes identificou uma barreira que impedia a aproximação da PSR nas UBS do município, barreira esta que se iniciava na própria recusa dos profissionais em atender esse 
público. Diante desta necessidade, a equipe de residência multiprofissional buscou criar um espaço de diálogo entre os profissionais da assistência social e saúde com a finalidade de garantir atendimento a PSR.

Assim, a organização do evento começou com uma reunião com a gestão do município, a Superintendente de Atenção Básica, a fim de pedir autorização e apoio para a realização do encontro. Após a anuência da gestão, foram enviados ofícios para cada UBS do município solicitando que estivessem presentes no encontro os ACS e enfermeiros (as).

Para o encontro foram selecionadas duas classes de profissionais das UBS pela importância de seus papéis nessa aproximação: os enfermeiros e os ACS. Os enfermeiros são os gerentes das UBS e têm contato com toda a equipe, além de serem responsáveis pela agenda de atendimentos nesses estabelecimentos, portanto, são peças-chave na melhoria do atendimento dessa população. Já os ACS são o elo entre a população e a UBS, sendo importantes na identificação das demandas daquele território. Portanto, seriam os principais responsáveis por identificar a PSR que necessitam de acompanhamento em suas áreas.

\subsection{ANÁLISE DOS DADOS}

Para nortear e melhor analisar os processos decorrentes do encontro, foram utilizados os referenciais teóricos relacionados à Política Nacional para a População em Situação de Rua, Política de Promoção de Equidade e portarias e lei relacionadas ao tema, tais como: Decreto Presidencial $\mathrm{n}^{\circ} 7.053$, de 23 de Dezembro de 2009, Portaria $\mathrm{n}^{0}$ 940, de 28 de abril de 2011, Lei $\mathrm{n}^{\circ} 8080$, de 19 de setembro de 1990.

\section{RESULTADOS E DISCUSSÃO}

O "I Encontro sobre as Políticas de Atendimento à População em Situação de Rua: Articulação entre SUS e SUAS" foi à primeira tentativa no município de Parnaíba-PI de promover uma discussão entre saúde e assistência social com foco na PSR, buscando apresentar a situação atual dessa população, os desafios e as políticas públicas voltadas para esse público, e assim despertar nos profissionais, público-alvo do evento, um novo olhar frente a essa população. Os principais tópicos e momentos de debate do evento são apresentados e discutidos a seguir.

\subsection{DIÁLOGO SOBRE POPULAÇÃO EM SITUAÇÃO DE RUA}

O município de Parnaíba, no que se refere à Atenção Básica, encontra-se dividido em 4 distritos. Como o público de ACS é numeroso, o encontro foi realizado em dias distintos para cada distrito, pois assim seria possível ter uma maior interação com o público presente. Já para os enfermeiros, o encontro foi realizado em dois dias distintos com a presença de dois distritos por dia.

O encontro com os ACS aconteceu na Escola de Gestão em Saúde Pública. Nele, um Professor Doutor da UFPI abordou temas como: "Quem é a população em situação de rua?", "Como vivem essas pessoas?", "Políticas públicas voltadas a este segmento da sociedade", "Fatores condicionantes", "A visão de rua na concepção dessa população" e "Os principais problemas de saúde que os acometem".

Já, o encontro com os enfermeiros aconteceu no mês de outubro de 2016, no Auditório da UBS do Bairro (CEEP). Nesta oportunidade, a equipe de profissionais do Centro POP foi responsável por abordar os temas já citados.

Desta forma, o termo "população em situação de rua" tem especificações heterogêneas, principalmente se comparadas às diversas realidades internacionais (ADORNO; WARANDA, 2004). No Brasil, o termo refere-se à situação do sujeito em relação à rua, mais do que somente a ausência de moradia. Desse modo, utilizaremos a definição segundo a qual a população em situação de rua consiste em um grupo populacional heterogêneo que tem em comum a pobreza extrema, os vínculos familiares interrompidos ou fragilizados e a inexistência de moradia convencional regular e que usa os logradouros públicos e as áreas degradadas como espaço de moradia e de sustento, de forma temporária ou permanente, bem como as unidades de acolhimento para pernoite temporário ou moradia provisória (BRASIL, 2009; BORYSOW; FURTADO, 2014). 
Este conceito é definido pela Política Nacional para a Pessoa em Situação de Rua no Decreto 7.053, de 23 de dezembro de 2009, que prediz como princípios a igualdade e equidade, o respeito à dignidade da pessoa humana, o direito à convivência familiar e comunitária, a valorização e respeito à vida e à cidadania, o atendimento humanizado e universalizado, o respeito às condições sociais e diferenças de origem, raça, idade, nacionalidade, gênero, orientação sexual e religiosa, com atenção especial às pessoas com deficiência (BRASIL, 2009; MATRACA; JORGE; WIMMER, 2014).

Pesquisa publicada pelo Ministério do Desenvolvimento Social no ano de 2008 traça um perfil do segmento populacional identificado como população em situação de rua, verificando que esta é predominantemente masculina (82\%), mais da metade tem entre 25 e 44 anos, com 53\%, e 67\% é constituída por negros. Apresenta níveis de renda baixos, sendo que a maioria $(52,6 \%)$ recebe entre $\$ \$ 20,00$ e $\$ \$ 80,00$ semanais (BRASIL, 2008; FIORATI et al., 2014).

Sobre os principais problemas de saúde desta população, o Instituto Meta, contratado pelo Ministério do Desenvolvimento Social e Combate à Fome (MDS) realizou uma pesquisa que apontou que $29,7 \%$ dos entrevistados afirmaram ter algum problema de saúde. Os problemas prevalentes foram: hipertensão $(10,1 \%)$, problemas psiquiátricos/mentais $\quad(6,1 \%), \quad$ HIV/AIDS $(5,1 \%)$, e problemas de visão/cegueira (4,6\%). Ademais, $18,7 \%$ dos entrevistados afirmaram fazer uso de algum medicamento e seu principal meio de acesso a eles são os postos/centros de saúde. Além disso, 43,8\% dos entrevistados afirmaram que procuram primeiramente o hospital/emergência quando estão doentes, e 27,4\% procuram o posto de saúde. Os locais mais usados pelas Pessoas em Situação de Rua para tomar banho são a rua (32,6\%), os albergues/abrigos (31,4\%), os banheiros públicos (14,2\%) e a casa de parentes ou amigos (5,2\%). Os locais mais usados pelas pessoas em situação de rua para fazer suas necessidades fisiológicas são a rua (32,5\%), os albergues/abrigos (25,2\%), os banheiros públicos $(21,3 \%)$, os estabelecimentos comerciais $(9,4 \%)$ e a casa de parentes ou amigos (2,7\%) (BRASIL, 2008, p.11).

Durante o evento, diversos dados foram discutidos, entre estes os relacionados à PSR com problemas de saúde. As equipes de saúde conheciam o caso, porém algumas relatavam não saber a forma como deveriam abordá-lo, enquanto outras levantaram questões relacionadas ao medo de conversar. Sobre estas questões, destaca-se que a PSR, historicamente, tem acesso limitado aos serviços de saúde, somando-se à existência de uma série de entraves para a efetivação do cuidado (SILVA; CRUZ; VARGAS, 2015; BARATA et al., 2015). Este dado corrobora com o estudo de Kamiet al. (2016) que destacou aspectos relacionados à resistência dos profissionais em atender e acolher a PSR.

Sobre este fato, encontra-se na literatura uma pesquisa nacional sobre a PSR que revela que $18,4 \%$ destas pessoas já passaram por experiências de impedimento de receber atendimento na rede de saúde (BRASIL, 2008). Embora, a Constituição Federal, em seu art. $2^{\circ}$, assegurar que a saúde é um direito fundamental do ser humano, devendo o Estado prover as condições indispensáveis ao seu pleno exercício (BRASIL, 1990), em termos resolutivos, não se observa a ampliação desses direitos à PSR, que vive à margem dos processos de inclusão e sofre graus acentuados de vulnerabilidade e marginalidade no acesso aos bens e serviços (CARNEIO; SILVEIRA, 2003; PAIVA et al., 2016).

Este também foi um momento de trocas de informações e experiências, pois a equipe de assistência social também conheceu a forma como as equipes de saúde trabalham e suas dificuldades. Tanto ACS quanto enfermeiros (as) puderam contribuir com suas colocações e dúvidas que eram inquietações da maioria, a saber: "Como abordar a pessoa em situação de rua? "Para onde encaminhá-la?" "Por que no estado existe somente um consultório na rua?". Nesse sentido, o trabalho multiprofissional é capaz de redesenhar a assistência e promover qualidade de vida ao usuário (SUGUYAMA; BUZZO; OLIVEIRA, 2016).

\subsection{DISPOSITIVO DA ASSISTÊNCIA SOCIAL DE MÉDIA COMPLEXIDADE- CENTRO POP}

Em um segundo momento do evento, a equipe do Centro POP apresentou o dispositivo da assistência social ao público presente, ressaltando quem são os usuários do serviço, sua localização, os objetivos, quais 
serviços são ofertados, estrutura, as formas de acesso e os profissionais de referência.

Verificou-se que muitos dos participantes desconheciam este dispositivo, alguns chegaram a questionar "Por que não houve este momento de compartilhamento de informações sobre o dispositivo anteriormente?" e "Os usuários dormiam no Centro POP?" e pediram contatos do serviço. Além disso, buscaram informações sobre como encaminhar pessoas em situação de rua para serem atendidas pelo Centro POP.

A equipe do Centro POP relatou que "mesmo havendo uma constante articulação do Centro POP com os dispositivos da saúde, ainda existem muitos problemas que, por vezes, inviabilizam o atendimento dos usuários", tendo em vista o próprio desconhecimento da população sobre este serviço no município.

Em tal caso, Trino, Machado, Rodrigues (2015) afirmam que, diante da complexidade de situações, de demandas e de necessidades que esta população tem, é imprescindível o investimento focado no cuidado compartilhado e implicado em rede.

Muito da discussão do tema desenvolveu-se em torno da necessidade de aproximar a Atenção Básica dessa população, bem como dos problemas de saúde mais frequentes nessa população e a problemática do Cartão Nacional de Saúde (Cartão do SUS). A falta deste documento acaba por barrar o atendimento de muitos usuários, que também não têm os documentos necessários para sua emissão (FARIAS et al., 2014).

A problemática do Cartão do SUS, de acordo com os relatos trazidos pela equipe de referência do Centro POP, é uma dificuldade habitual em relação ao atendimento à saúde, principalmente quando há necessidade de marcação de exames, pois muitas das vezes o usuário não possui documentos necessários solicitados pelos dispositivos da saúde.

A Portaria de $\mathrm{n}^{\circ}$ 940, de 28 de abril de 2011, que regulamenta o Sistema Cartão Nacional de Saúde (Sistema Cartão), no $\S 1^{\circ}$ artigo 23, dispensa aos ciganos, nômades e moradores de rua a exigência de apresentar o endereço do domicílio permanente para aquisição do Cartão SUS. Desse modo, é importante enfatizar que qualquer pessoa tem o direito de ser atendido nas unidades de saúde, independente de apresentação de documentação (BRASIL, 2011; HALLAIS; BARROS, 2015).
Verifica-se que é necessária uma flexibilização do que é preconizado por esta portaria, tendo em vista que muitos usuários não possuem cartão SUS e tampouco endereço fixo, sendo informações solicitadas na marcação de consultas e exames.

Dada a complexidade das situações abordadas, um ponto a ser pontuado futuramente é as rodas de conversas com os gestores locais, tendo como tema o cadastramento e as informações sobre a população em situação de rua (principalmente no que concerne às necessidades de saúde), as dificuldades relacionadas ao acesso deste segmento da população aos serviços de saúde e assistência social e a solicitação de investimentos na implantação (Consultório na Rua) e qualificação da redes de atendimento à população em situação de rua.

\subsection{DISCUTINDO O CUIDADO- ESTUDOS DE CASOS}

O término do Encontro deu-se com a metodologia de estudos de casos baseados na resolução de problemas. Apresentaram aos participantes narrativas reais que continham uma situação problema. Estes casos foram selecionados aleatoriamente dentre aqueles que haviam sido acompanhados pela equipe do Centro POP. Eles são de ampla complexidade e envolvem questões pertencentes ao campo da saúde, como otite, doenças transmissíveis, problemas de pele, entre outros.

Diante deste cenário, verifica-se que inúmeros são os desafios para diminuir as iniquidades em saúde da População em Situação de Rua, desafios estes relacionados a problemas de enorme multiplicidade, demandas e necessidades, tais como: os agravos e doenças como o alcoolismo e a dependência a outras drogas, doenças mentais e diversas formas de sofrimento psíquico, causadas, em sua maioria, pela perda ou precarização dos laços familiares e sociais e da própria identidade social, DST's, AIDS, tuberculose e doenças dermatológicas (AGUIAR; IRIAT, 2012; BRASIL, 2013; MACERATA; SOARES; RAMOS, 2014).

Assim, durante a discussão desses casos, os profissionais reuniam-se em grupos e tinham a responsabilidade de desenvolver uma estratégia de intervenção para cada caso proposto em conjunto com suas equipes da ESF de cada UBS. Após a discussão em 
equipe, seguia uma apresentação na qual cada equipe escolhia um relator que iria ler o caso para todos os presentes e expor as possíveis intervenções. Após o relato das equipes sobre as possíveis soluções para os casos, a equipe do Centro POP relatou a forma como havia lidado com cada caso, afirmando que não necessariamente seria a mais adequada, porém era a viável naquele momento com o uso ferramentas que tinham a sua disposição.

$\mathrm{Na}$ apresentação, algumas equipes enfatizaram a construção de redes de cuidado entre Atenção Básica, Centro POP, os Centros de Atenção Psicossocial, sendo imprescindível o investimento no cuidado compartilhado. Neste contexto a necessidade de articulação intersetorial entre trabalhadores de diversas áreas que atuam com a PSR é fundamental, particularmente entre as equipes da assistência social e saúde (BRASIL, 2015). As ações intersetoriais constituem-se em uma prerrogativa necessária para responder às necessidades da PSR (SERAFINO; LUZ, 2015).

Por fim, foram debatidas possíveis articulações que poderiam ser realizadas dentro da rede de saúde e assistência social no intuito de resolver aqueles problemas.

\subsection{DESAFIOS PARA O CUIDADO DA SAÚDE DA PSR}

No decorrer do encontro, foram apontados alguns desafios pelos participantes no que tange à maneira de lidar com este tema, principalmente sobre como abordar a PSR, os medos inerentes a esta prática de cuidado a saúde e a falta de qualificação profissional para atuar com a população em situação de rua.

Alguns relataram que é "necessário para realizar a abordagem e acolhimento do usuário um momento de qualificação dos profissionais, tendo em vista ser algo novo para os mesmos".

Sobre esta questão, Trino, Machado, Rodrigues (2015, p. 28) citam que:

Investir na qualificação das equipes, e no fortalecimento das redes intersetoriais. Qualificar os serviços tendo como diretriz o acolhimento, incidindo não apenas nas relações das equipes com as pessoas em situação de rua, mas também entre estes e os demais usuários dos serviços.
Perante aos desafios apontados anteriormente, não podemos deixar de fazer menção algumas mudanças no campo da saúde, principalmente após a implementação da Política de Promoção de Equidade pelo Ministério da Saúde. Tal política visa legitimar os direitos de muitas pessoas que historicamente, sempre fizeram parte de populações estigmatizadas e discriminadas, cita-se aqui a PSR.

A partir do momento em que se cria espaços de debates como os do "Encontro", possibilita-se a sensibilização dos setores envolvidos (saúde e assistência) e, assim, a responsabilização pelos casos, construindo metodologias e práticas de cuidado mais humanizadas.

Observa-se alguns avanços, a exemplo da Política de Promoção de Equidade. Esta tem como objetivo diminuir as vulnerabilidades a que certos grupos populacionais estão mais expostos e que resultam de determinantes sociais da saúde, como os níveis de escolaridade e de renda, as condições de habitação, acesso à água e saneamento, à segurança alimentar e nutricional, a participação da política local, os conflitos interculturais e preconceitos com o racismo, as homofobias e o machismo, entre outros (BRASIL, 2013, p. 06).

Contudo, mesmo que se tenha observado algumas mudanças neste âmbito, percebe-se ainda as dificuldades de acesso ao SUS por este segmento da população. Faz-se necessário o compartilhamento do cuidado localmente, no território, investindo na articulação e fortalecimento das redes intersetoriais (TRINO; MACHADO; RODRIGUES, 2015).

\section{CONCLUSÃO}

O "I Encontro sobre as Políticas de Atendimento a Pessoa em situação de Rua: Articulação entre SUS e SUAS" foi um espaço de troca em que atores da saúde e assistência social puderam conhecer, discutir e refletir de forma crítica sobre o cuidado em saúde da população em situação de rua. O evento não contou com instrumento de avaliação sistemática, deste modo, apresenta-se como sugestão para as futuras ações coletivas.

As dificuldades elencadas pelos profissionais quanto ao atendimento da pessoa em situação de rua, 
tais como "dificuldades de acesso", "falta do cartão SUS" e "resistências em abordar a PSR," é um desafio de todos os setores da sociedade, que é responsável pelas construções de estratégias de resolubilidade no cuidado compartilhado, destacando-se a necessidade de superação do preconceito e o investimento na qualificação das equipes. Por fim, tem-se a expectativa de que momentos como estes gerem avanços nas discussões sobre cuidado à saúde da PSR, abrindo espaços para diálogo entre os profissionais e rodas de conversas com os gestores locais.

\section{REFERÊNCIAS}

ADORNO, R. C. F.; VARANDA, W. Descartáveis urbanos: discutindo a complexidade da população de rua e o desafio para políticas de saúde. Saúde e Sociedade, v. 13, n. 1, p. 23-45, 2004.

AGUIAR, M. M.; IRIART, A. B. Significados e práticas de saúde e doença entre a população em situação de rua em Salvador, Bahia, Brasil. Cad. Saúde Pública, v.28, n.1, n.115-124, 2012.

BARATA, R. B. Desigualdade social em saúde na população em situação de rua na cidade de São Paulo. Saúde Soc. v.24, supl.1, p.219-232, 2015.

BORYSOW, I. C.; FURTADO, J. P. Acesso, equidade e coesão social: avaliação de estratégias intersetoriais para a população em situação de rua. RevEscEnferm USP, v. 48, n. 6, p.1069-76, 2014.

BRASIL, Ministério da Saúde. Políticas de Promoção das Equidades em Saúde. Brasília, 2013.

BRASIL, Ministério do Desenvolvimento Social e Combate à Fome. Perguntas e Respostas: Centro de Referência Especializado para População em Situação de Rua-Centro POP. Brasília, 2011.

BRASIL. Decreto Presidencial $\mathrm{n}^{\circ} 7.053$, de 23 de Dezembro de 2009. Institui a Política Nacional para a População em Situação de Rua e seu Comitê Intersetorial de Acompanhamento e Monitoramento, e dá outras providências. Diário Oficial da União, Brasília, DF, Seção 1, 24 dez. 2009.
BRASIL. Lei $\mathrm{n}^{\mathrm{o}}$ 8080, de 19 de setembro de 1990. Dispõe sobre as condições para a promoção, proteção e recuperação da saúde, a organização e o funcionamento dos serviços correspondentes e dá outras providências. Diário Oficial da União, Brasília, DF, 19 set. 1990.

BRASIL. Ministério da Saúde. Portaria no 940/GM/MS, 28 de abril de 2011. Regulamenta o Sistema Cartão de Saúde (Sistema Cartão). Diário Oficial da União, Brasília, DF, Sessão 1, 29 de abr. 2011.

BRASIL. Ministério da Saúde. Saúde da população em situação de rua: um direito humano. Brasília-DF, 2015.

BRASIL. Ministério do Desenvolvimento Social e Combate à Fome. Pesquisa nacional sobre a população em situação de rua. Brasília, 2008.

CARNEIRO, J.N.; SILVEIRA, C. Organização das práticas de atenção primária em saúde no contexto dos processos de exclusão/inclusão social. Cad Saúde Pública, v. 19, v. 6, p. $1827-35,2003$.

FARIAS, D. C. S. et al. Saberes sobre saúde entre pessoas vivendo em situação de rua. Psicologia e Saber Social, v.3, n.1, p. 70-82, 2014.

FIORATI, R. C. População em vulnerabilidade, intersetorialidade e cidadania: articulando saberes e ações. Saúde Soc, v.23, n.4, p.1458-1470, 2014.

HALLAIS, J. A. S.; BARROS, N. F. Consultório na Rua: visibilidades, invisibilidades e hipervisibilidade. Cad. Saúde Pública, v.31, n.7, p.1497-1504, 2015.

KAMI M. T. M. et al. Saberes ideológicos e instrumentais no processo de trabalho no Consultório na Rua. Rev Esc Enferm USP, v. 50, n. 3, p.442-449, 2016.

MARCERATA, I.; SOARES, J. G. N.; RAMOS, J. F. C. Apoio como cuidado de territórios existenciais: Atenção Básica e a rua. Interface comunicação saúde educação, v.18, Supl. 1, n.919-30, 2014.

MATRACA, M. V. C.; JORGE, T. C. A; WIMMER, G. O PalhaSUS e a Saúde em Movimento nas Ruas: relato de um encontro. Interface comunicação saúde educação, v.18, Supl. 2, p.1529-1536, 2014. 
PAIVA, I. K. S. et al. Direito à saúde da população em situação de rua: reflexões sobre a problemática. Ciênc. saúde coletiva, v.21 n.8, p. 2595- 2606, 2016.

SERAFINO, I; LUZ, L. C. X. Políticas para a população adulta em situação de rua: questões para debate. Revista Katálysis, v. 18, n. 1, p. 74-85, 2015.

SILVA, C. C.; CRUZ, M. M.; VARGAS, E. P. Práticas de cuidado e população em situação de rua: o caso do Consultório na Rua. Saúde Debate, v. 39, n. Especial, p. 246-256, 2015.

SUGUYANA, P.; BUZZO, L. E.; OLIVEIRA, M. L. F. Desvelando a vivência da equipe multiprofissional no cuidar do paciente esquizofrênico. Saúde e Pesquisa, v. 9, n. 1, p. 65-71, 2016.

TRINO, A. T.; MACHADO, M. P. M.; RODRIGUES, R. B.Conceitos norteadores do cuidado junto à população em situação de rua. In: TEIXEIRA, M.; FONSECA, Z. Saberes e práticas na atenção primária à saúde: cuidado à população em situação de rua e usuários de álcool, crack e outras drogas. São Paulo: Hucitec, 2015. p. 27-53.

Recebido em: 24 de maio de 2017 Aceito em: 01 de agosto de 2017 